Bangladesh J. Bot. 44(4): 543-549, 2015 (December)

\title{
MS-DELAYED LIGHT EMISSION (MS-DLE) OF CHLOROPHYLL AS AN INDICATOR OF TEMPERATURE STRESS ACTION ON PHOTOSYSTEM II (PS II)
}

\author{
Ali Bashirzadeh $^{*}$ Zaman Mahmudov $^{1}$ and Ralphreed Hasanov ${ }^{1}$ \\ Department of Agriculture, Islamic Azad University, Astara Branch, Iran
}

Key words: Photosystem II (PSII), MS-DLE, Photoinhibition, Temperature stress

\begin{abstract}
Action sites of low positive temperature together with high light intensities in electron transport reactions of photosystem II (PSII) evaluated by ms range delayed fluorescence (ms-DLE) of chlorophyll a content in the maize and barley seedlings are presented. The main targets for these stress factors action were shown to be $\mathrm{Y}_{\mathrm{z}}$ and $\mathrm{Mn}_{4} \mathrm{O}_{\mathrm{x}} \mathrm{Ca}$-cluster on the donor site of PSII in the case of simultaneous action of different temperatures and high light illumination and between $\mathrm{Q}_{\mathrm{A}}$ and $\mathrm{Q}_{B}$ on the acceptor site of PSII in the case of low positive temperature influence only.
\end{abstract}

\section{Introduction}

It was identified that photosystem II (PSII) is the most sensitive to the changes of environmental conditions and under the influence of these unfavorable factors of PSII activity is more affected, than the activities of other physiological processes (Tyystjärvi 2013). In earlier investigations, the major attention was given to the explanation of PSII problems in vitro damage mechanisms under the influence of various stress factors (Álvarez et al. 2012). It was determined that high temperature inactivates the chloroplasts and at first the reactions connected with oxygen evolution. Oxygen evolution is inactivated under the incubation of chloroplast thylakoids at 42 $45^{\circ} \mathrm{C}$ within several minutes. Higher concentrations of saccharose increase the thermo stability of oxygen evolution as the result of stabilization of $33 \mathrm{kDa}$ protein connection with the PSII nucleus (Tyystjärvi 2008, Allakhverdiev et al. 2008). PSII reactions is most sensitive different stresses like low temperature $\left(0-14^{0} \mathrm{C}\right)$ or high light intensity. Species those are sensitive to low temperatures have a tendency to display photoinhibition of PSII when they are exposed to high light intensity under low temperatures (Duffy et al. 2013). Low temperature can reduce photosynthetic capacity of photosystem II, turnover reactions at low temperatures are diminished, and low temperature can reduce capacity of the reactive oxygen species scavengers, which can provide protection against photoinhibition in vivo (Liu et al. 2013). Arising mainly from in vitro studies, two mechanisms of photoinhibition of PSII have been proposed: Acceptor-side and donor-side photoinhibition (Allakhverdiev et al. 2011).

\section{Materials and Methods}

The kinetics of ms-DLE was measured using a phosphoroscope as described previously $(16 ; 9)$. A sample in silica-suprasil cuvette was irradiated with continuous white light $(250$ $\mathrm{W} / \mathrm{m}^{2} \mathrm{~s}$ ) passing through a $2 \mathrm{~cm}$ of a $\mathrm{CuSO}_{4}$ solution. The light was passed through holes on the rotating wheel of the phosphoroscope (three holes, $120^{\circ}$ apart) in such a way that $0.3 \mathrm{~ms}$ of excitation was following by $1.25 \mathrm{~ms}$ of dark and $0.3 \mathrm{~ms}$ of registration of the delayed light

\footnotetext{
*Author for correspondence. <a.bashirzadeh@iau-astara.ac.ir>.

${ }^{1}$ Department of Biophysics and Molecular Biology, Baku State University, Z. Khalilov str.23, Baku, AZ1148 Azerbaijan.
} 
emission (three cycles) of excitation/dark/registration per one full turnover of the phosphoroscope wheel. The analyses were run in 3-replications for each variants of measurement on 4-6 standard leaflets. Barley (Hordeum vulgare L. cv. Makooi Barle) and maize (Zea mays L. cv. Var. 540) seedlings were grown within seeds soaked at $25^{\circ} \mathrm{C}$ for 7 days under the artificial light $(600 \mu \mathrm{mol}$ photons $/ \mathrm{m}^{2} \mathrm{~s}$ within 16 hrs by 8 hourly dark interval) were used. Photoinhibition treatment of leaves of barley and maize seedlings was given to a laboratory built in a specially designed chamber with constant temperature, air and humidity as described earlier (Mahmudov et al. 2005). Treatment of leaves of barley and maize seedlings by different stresses was given in a laboratory built in a specially designed chamber with regulation of temperature, air and humidity conditions (Mahmudov et al. 2005). Leaves of seedlings were exposed in a chamber with selected low or high temperatures and illumination with artificial lights $\left(2400 \mu \mathrm{mol}\right.$ photons $\left./ \mathrm{m}^{2} / \mathrm{s}\right)$ for $5-60 \mathrm{~min}$.

The analyses were run in three-replications for each variant of measurement on 4 - 6 standard leaflets. F-fast component-reflects a reduction of first acceptors; transition of RCII to the closed state; a state of reaction centers of PSII; SP-slow component-reflects a chemical components of photochemical gradient of protons and a change of electron transport rate. Fast phase (FP) reflects a reduction of the first acceptors and transition of reaction centers to (RCII) the closed state; and in general the a state of reaction centers of PSII; slow phase (SP)-reflects chemical phases of photochemical gradient of protons and change of electron transport rate and in general state of donor side of PSII. Results are expressed as \pm mean Sd for a given number of observations $(\mathrm{n} \geq$ 3). The level of significance was set at $\rho$ value of 0.05 . Microsoft Office Excel 2010 programme was used for figures preparation and stastistic evaluation were calculated by Sigma Plot programe.

\section{Results and Discussion}

Fig. 1 shows that there is a great deal of variation between the two curves caused by ms-DLE among maize seedlings under $4^{\circ} \mathrm{C}$ and $24^{\circ} \mathrm{C}$ this variation, especially the second slow phase of (ms-DLE), represents itself apparently as an indicator among maize seedlings. Therefore, the $\mathrm{SP}_{1}$ exsistant in ms-DLE has developed at a temperature of $4^{\circ} \mathrm{C}$ versus $24^{\circ} \mathrm{C}$ that is easily noticed.

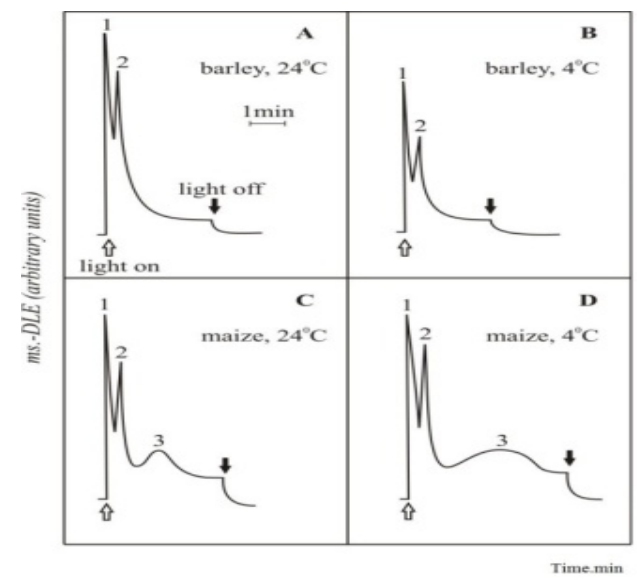

Fig. 1. Protocols of measurements of the ms-DLE induction curves of barley and maize seedlings at $24^{\circ} \mathrm{C}$ and at cool conditions $\left(4^{\circ} \mathrm{C}\right) ; 1$ - DLEFP; 2 - DLE SP; 3 - DLE SP ${ }_{1} ; 4$ - DLE SS.

Ms-DLE kinetic parameter intensity of maize seedlings reduced meaningfully at $4^{\circ} \mathrm{C}$ (Fig. 1). Special changes were observed regarding induction kinetic curves maize seedlings. Therefore, $\mathrm{SP}_{1}$ 
seen among maize seedlings at $4^{\circ} \mathrm{C}$ increased the kinetic resistance duration two times (Fig. 1 A). $\mathrm{SP}_{1}$ formation completed in a minute at $24^{\circ} \mathrm{C}$ while it took two minutes at $4^{\circ} \mathrm{C}$.

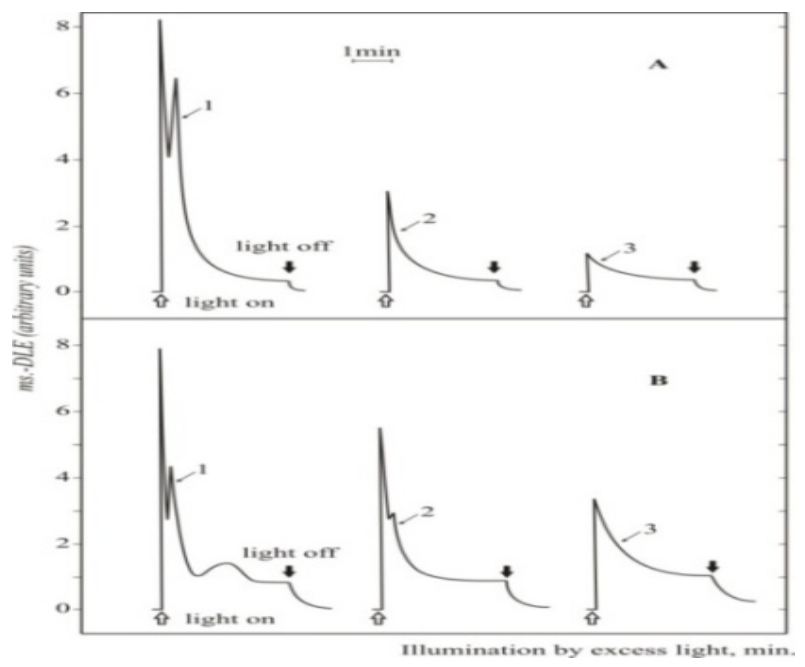

Fig. 2. Changes of ms-DLE induction curves after photoinhibition $\left(2400 \mu\right.$ molphotons $\left./ \mathrm{m}^{2} \mathrm{~s}\right)$ at definite designated time at $24^{\circ} \mathrm{C}$ of the barley (A) and maize (B). A: 1 - no illumination; 2 - 5 min illuminition; 3 - 20 min illuminition; B: 1 - no illumination; 2 - 5 min illumination; 3-15 min illuminition.

Maize and barley seedlings are completely dependent on incubation (duration) factor at low favorite temperature. $\mathrm{SP}_{1}$ was the only phase among maize seedlings that tended to have positive reaction to low positive temperature (Fig. 2). It seems that seedling incubation findings under $14^{\circ} \mathrm{C}$ showed a small reduction in fast phase intensity even among barley seedlings, after a 5 minute period, the low positive temperature effect and FP intensity of $\mathrm{Chl}_{\mathrm{a}}$ ms-DLE induction curves decreased considerably $(75 \%)$.

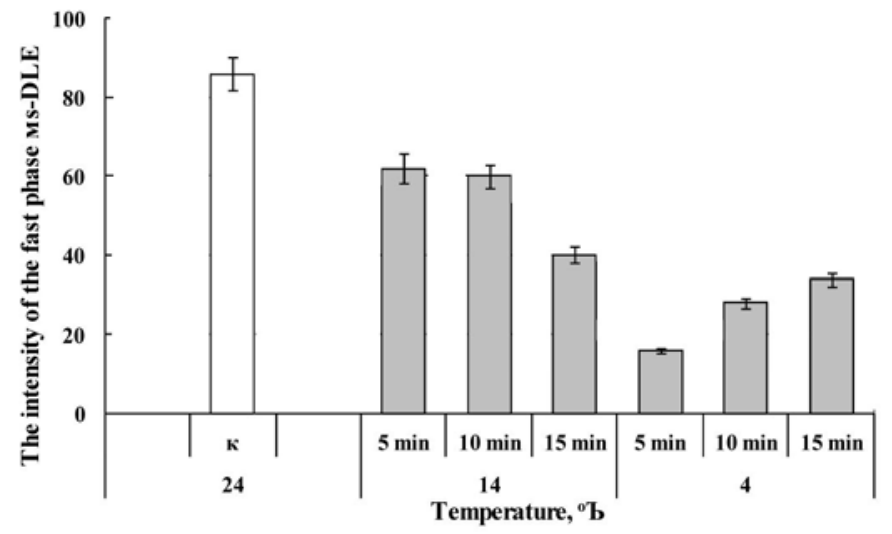

Fig. 3. FP intensity of $\mathrm{Chl}_{\mathrm{a}}$ ms-DLE induction curves among barley seedlings at low temperatures based on different preservation periods. 


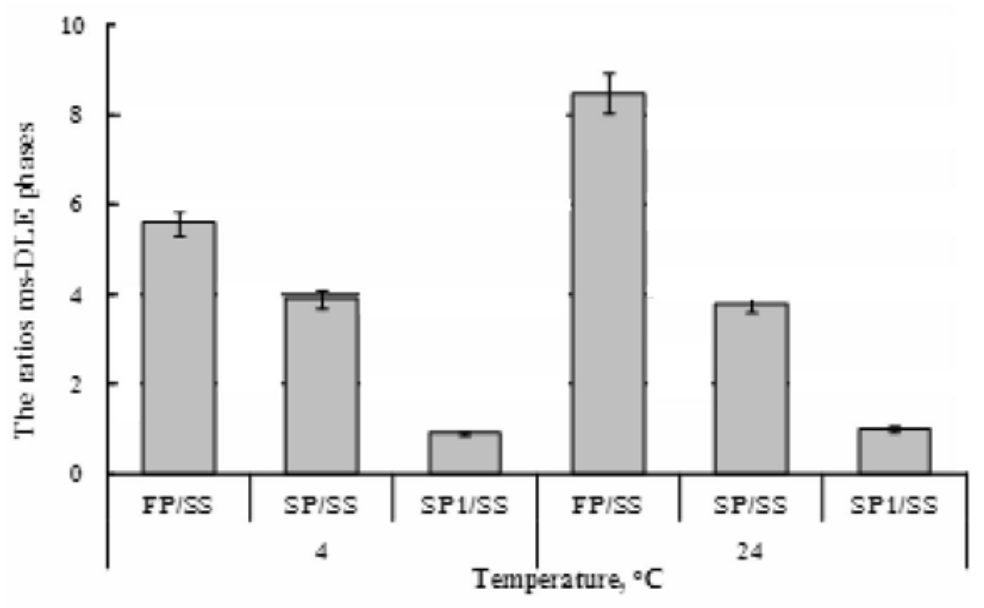

Fig. 4. Changes of different phase ( $\left.\mathrm{Ch} \mathrm{a}_{\mathrm{a}} \mathrm{ms}-\mathrm{DLE}\right)$ among maize seedlings about SS dependent on temperature changes during preservation period for 20 minutes.

As figure 4 shows, the numeral value of FP ratio and $\mathrm{SP}_{1}$ decreased significantly. SP intensity of $\mathrm{Chl}_{\mathrm{a}}$ ms -DLE induction curve was completely stable and the experimental version of maize seedlings at $24^{\mathrm{C}^{\circ}}$ remained actually unchanged versus 20 minutes preservation period at low positive temperature during incubation. Considering the effects at $4^{\mathrm{C}^{\circ}}$ lasting for 20 minutes, changes at FP, SP and SS versus in vitro control at $24^{\mathrm{C}^{\circ}}$ led to interesting conclusions (Fig. 4).

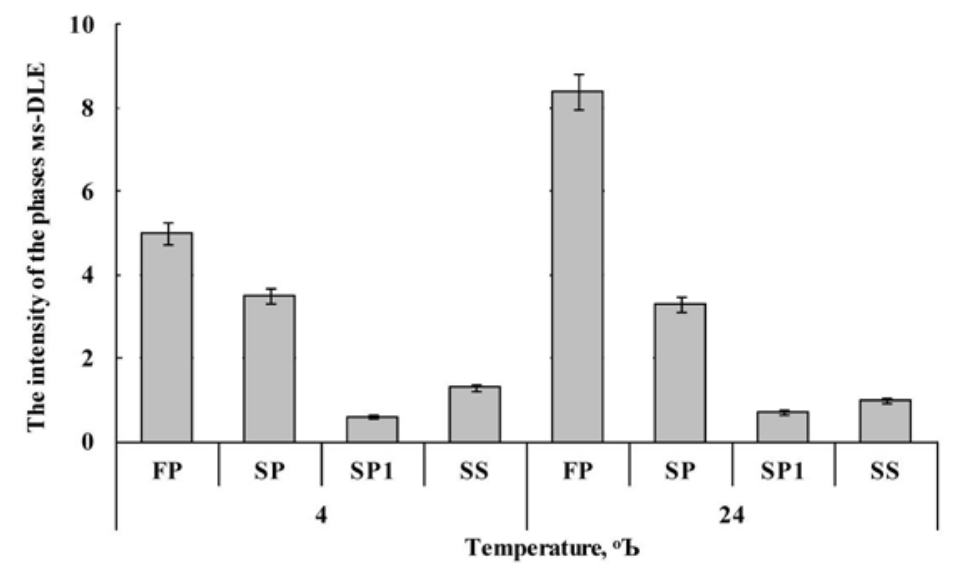

Fig. 5. Changes in development of different phases of $\mathrm{Chl}_{\mathrm{a}}$ ms-DLE induction curves among maize seedlings dependent on temperature changes during preservation period for 20 minutes.

It seems, according to induction curves, the persistence of $\mathrm{SP}_{1}$ increased more than two times at the temperature of $4^{\circ} \mathrm{C}$. In addition, the SS increase existent in $\mathrm{Chl}_{\mathrm{a}}$ ms-DLE induction curves was seen among maize seedlings (Fig. 5). The intensity of $\mathrm{SP}_{1}$ of decreased slightly and, within SS intensity, the radiation from $\mathrm{Chl}_{\mathrm{a}}$ ms-DLE induction curve significant reduction was observed. Therefore, like barley seedlings, low positive temperatures influenced significantly the nature of $\mathrm{Chl}_{\mathrm{a}}$ ms-DLE induction curve among maize seedlings. Changes in the different phases ms-DLE 
induction curves at different illumination time by excess light for barley and maize seedlings at 24 and $4^{\circ} \mathrm{C}$ are shown in Fig. 6, A and B, respectively. As expected, there was an inhibition of the $\mathrm{FP}, \mathrm{SP}$ and $\mathrm{SP}_{1}$ phases, and increasing of SS level by illumination with excess light. This photoinhibition is observed more clearly in leaves of maize seedling (Fig. 6 B) if it is compared to leaves of barley seedlings.

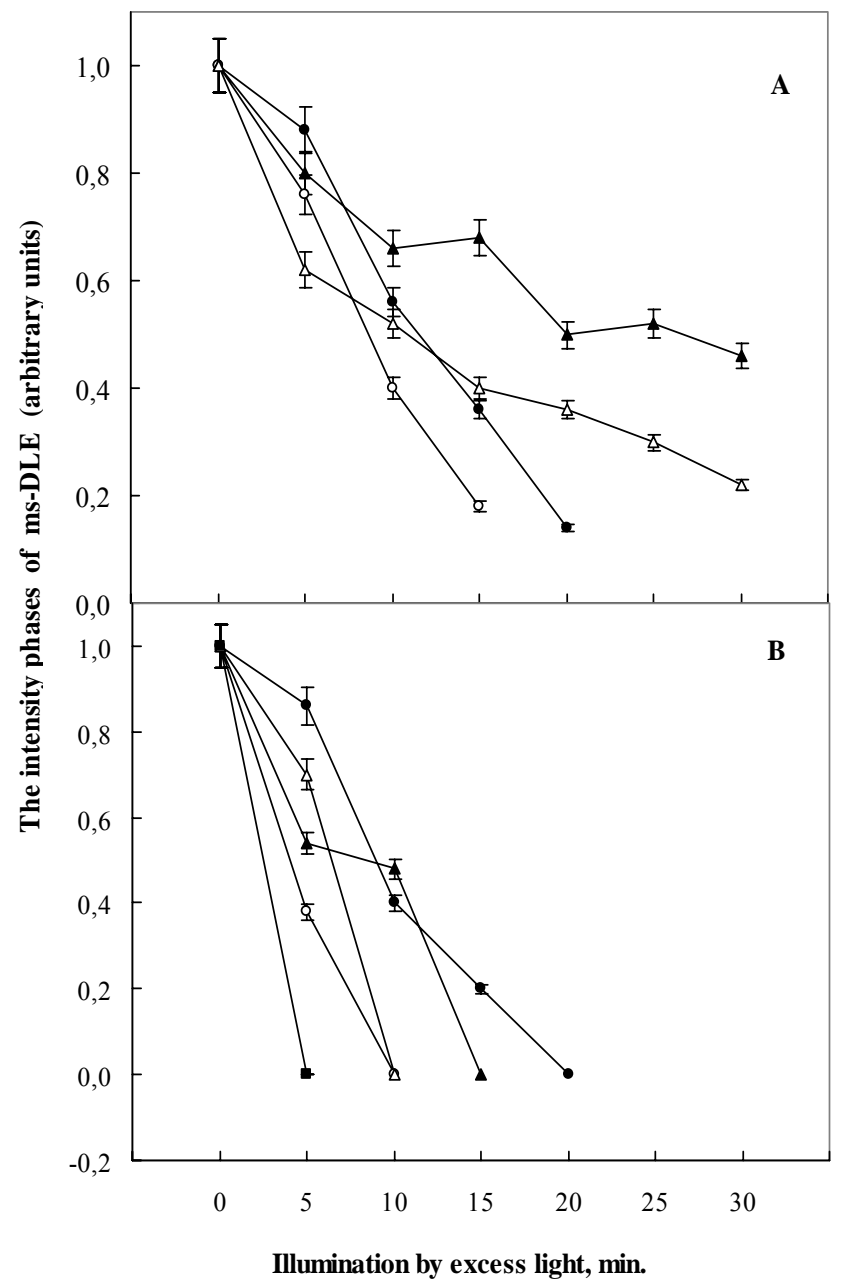

Fig. 6. ms-DLE components as a function of illumination time by excess white light for barley and maize. A: DLEFP $\Delta$-maize, $4^{\circ} \mathrm{C} ; \boldsymbol{\Delta}$-maize, $24^{\circ} \mathrm{C}$; o-barley, $4^{\circ} \mathrm{C} ; \bullet$-barley, $24^{\circ} \mathrm{C}$.B: DLESP $\Delta$-maize, $4^{\circ} \mathrm{C} ; \boldsymbol{\Delta}$-maize, $24^{\circ} \mathrm{C}$; ○-barley, $4^{\circ} \mathrm{C}$; •-barley, $24^{\circ} \mathrm{C}$; DLESP 1 -maize, $24^{\circ} \mathrm{C} ; \square$-maize, $4^{\circ} \mathrm{C}$.

The result in Fig. 6 shows that both tensions (extreme temperature as well as high light intensity) damage the sites adjacent to electron donor side in PSII. So, with the help of measuring and analyses of induction curve of ms-DLE chlorophyll $a$ in barley and maize leave seedlings attempt to demonstrate the damage sites of PSII under the influence of low positive temperature and high intensity of visible light. The method of delayed light emission of chlorophyll $a$ from 
PSII, particularly the slow phase change induction curve of ms-DLE gives an opportunity to indicate, whether undergoes inactivation of known side of PSII, probably $\mathrm{Mn}_{4} \mathrm{O}_{\mathrm{x}}$ Ca-cluster of PSII. As a rule, depending on surrounding partner for recombination reactions of reaction of $\mathrm{P} 680 \mathrm{Q}_{\mathrm{A}}{ }^{-}$with one of the states of $S^{\prime}$, of the water oxidizing reactions of photo- oxidation of $\mathrm{Mn}_{4} \mathrm{O}_{\mathrm{x}} \mathrm{Ca}$-cluster (Vass et al. 1992). It was known that ms-DLE may be attributed to stabilization reactions at the donor side of PSII (Allakhverdiev et al. 2008). Recently, it was shown that msDLE originates from $\mathrm{Y}_{\mathrm{Z}}^{+}{ }^{*} \mathrm{P} 680 \mathrm{Q}_{\mathrm{A}}{ }^{-}$state via radiation recombination (Oukarroum et al. 2013). The ms-DLE SP phase represents this state being in equilibrium with the S-states of the water oxidizing complex (Gasanov et al. 2007). FP, functioning as the indicator of electron director in PSII, didn't show considerable change in low positive temperature tension condition. However, ms-DLE-SS increased. It is probable that present situation is the result of special changes around $\mathrm{Q}_{\mathrm{A}}-\mathrm{Q}_{\mathrm{B}}$ sites. Decay to the SS level reflects disappearance of charge separated state due to the following electron transfer from $\mathrm{Q}_{\mathrm{A}}{ }^{-}$on the acceptor side of PSII (Lavorel 1975). The result showed that low positive temperature $\left(4^{\circ}-14^{\circ}\right)$ along with high light intensity and short-term effect in different periods having different intensities and ms-DLE induction curves cause significant reduction of both phase; ms-DLE-FP and ms-DLE-SP (Fig. 2). It shows that in low temperature and high light intensity condition there is a possibility that $\mathrm{Chl}_{\mathrm{a}}$ ms-DLE in PSII that is the recombination of $\mathrm{Tyr}_{\mathrm{Z}}$ and $\mathrm{Mn}_{4} \mathrm{O}_{\mathrm{x}} \mathrm{Ca}$ indicator, is damaged by forming oxidation. Charge recombination reaction in PSII at high intensity of light and low temperature is much slower and in some conditions even never takes place and SS is increased (Bertamini et al. 2007, Feng et al. 2005).

The case indicates that damaged sites of the two tensions are located at separate parts. The results show that under low positive temperature in PSII the electron acceptor side, i.e, the area around $\mathrm{Q}_{\mathrm{A}}-\mathrm{Q}_{\mathrm{B}}$ sites and high intensity light in electron donor side in PSII ,i.e. the area around $\mathrm{Tyr}_{\mathrm{Z}}$ and $\mathrm{Mn}_{4} \mathrm{O}_{\mathrm{x}} \mathrm{Ca}$ sites faced damage (Feng et al. 2005, Vass 2012).

\section{References}

Allakhverdiev SI, Kreslavski VD, Klimov VV, Los DA, Carpentier R and Mohanty P 2008. Heat stress: An overview of molecular responses in photosynthesis. Photosynth. Res. 98: 541-550 .

Allakhverdiev SI, Tsuchiya T, Watabe K, Kojima A, Los DA, Tomo T, Klimov VV and Mimuro M 2011. Redox potentials of primary electron acceptor quinone molecule $\left(\mathrm{Q}_{\mathrm{A}}{ }^{-}\right)$and coserved energetics of photosystemII in cyanobacteria with chlorophyll $a$ and chlorophyll $d$. Proc. Natl. Acad. Sci. U.S.A. 108, 8054-8058.

Álvarez C, Sáez P, Sánchez-Olate M and Ríos D 2012. Effects of light and ventilation on physiological parameters during in vitro acclimatization of Gevuina avellana Mol. Plant Cell Tissue Organ Cult. 108: 1-9 .

Baker NR 2008. Chlorophyll fluorescence : A probe of photosynthesis in vivo - Annu. Rev. Plant Biol. 59: 89-113.

Bertamini M, Zulini L, Zorer R, Muthuchelian K and Nedunchezhian N 2007. Photoinhibition of photosynthesis in water deficit leaves of grapevine (Vitis vinifera L.) plants. Photosynthetica 45: 426-432.

Duffy CD, Valkunas L and Ruban AV 2013. Light-harvesting processes in the dynamic photosynthetic-antenna. Phys. Chem. 15: 18752-18770.

Feng YL and Cao KF 2005. Photosynthesis and photoinhibition after night chilling in seedlings of two tropical three species grown under three irradiances. Photosyntetica. 43: 567-574.

Gasanov R, Aliyeva S, Arao S, İsmailova A, Katsuta N, Kitade A, Yamada S, Kavamori A and Mamedov F 2007. Comparative study of the water oxidizing reactions and the millisecond delayed chlorophyll fluorescence in photosystem II at different $\mathrm{pH}$. Photochem. Photobiol. B: Biology 86: 160-164.

Lavorel J 1975. Luminescence. - In: Govindjee (ed.): Bioenergetics of photosynthesis, Academic Press pp. 225-319.

Liu W, Yu K, He T, Li F, Zhang D and Liu J 2013. The low temperature induced physiological responses of Avena nuda L., a cold-tolerant plant species. The Scientific World 2013: 1-7.

Mahmudov ZM, Abdullayev KhD, Gasanov RA 2005. Phohoinhibition in vivo of photosystem-II reaction 
during development of the photosystem II at different $\mathrm{pH}$. J. Photosystems of wheat seedlings. Photosynth. Res. 84: 9-14.

Oukarroum A, Goltsev V, Strasser RJ 2013. Temperature effects on pea plants probed by simultaneous measurements of the kinetics of prompt fluorescence, delayed fluorescence and modulated $820 \mathrm{~nm}$ reflection. PLoS One 8: e59433.

Tyystjärvi E 2008. Photoinhibition of PhotosystemII and photodamage of the oxygen-evolving manganese cluster". Coordination Chemistry Reviews 252: 361-376.

Tyystjärvi E 2013. Photoinhibition of photosystem II. Int. Rev. Cell Mol. Biol. 300: 243-303.

Vass I 2012. Molecular mechanisms of photodamage in the photosystem II complex. Biochim Biophys Acta. 1817: 209-217.

Vass I, Styring S, Hundal T, Koivuniemi A, Aro EM and Andersson B 1992. Reversible and irreversible intermediates during photoinhibition of photosystem II: Stable reduced $\mathrm{Q}_{\mathrm{A}}$ species promote chlorophyll triplet formation. - Proc. Natl. Acad Sci USA 89: 1408-1412.

(Manuscript received on 13 March, 2015; revised on 29 October, 2015) 